\title{
Peroral endoscopic pyloromyotomy for primary pyloric stenosis
}

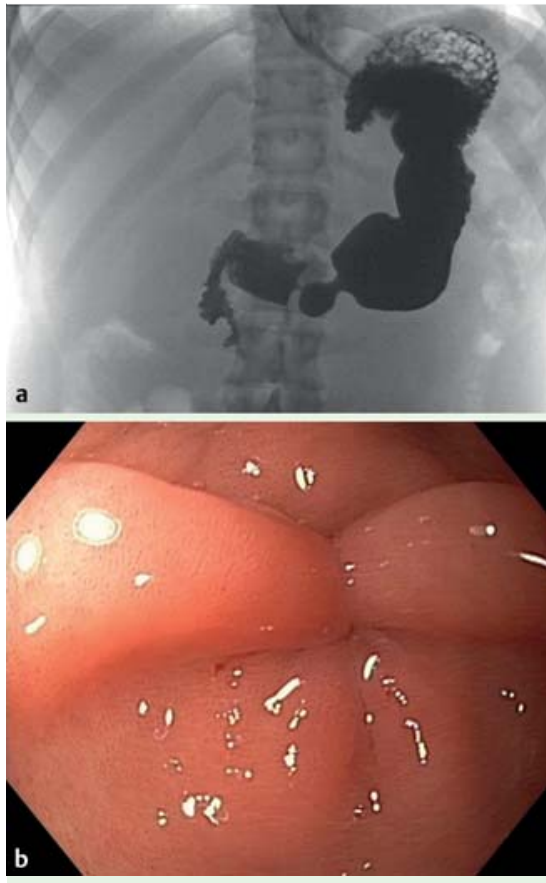

Fig. 1 a Marked delay in the passage of contrast through the pylorus is observed during dynamic gastrography in an 18-year-old man with adult idiopathic hypertrophic pyloric stenosis. $\mathbf{b}$ Before pyloromyotomy, there is considerable resistance to passage of the endoscope through the pylorus.

Primary pyloric stenosis (PPS) in adults is a rare condition. The symptoms are those of gastric outlet obstruction. The standard treatment is balloon dilation, pyloroplasty, or occasionally surgical distal gastrectomy [1]. Peroral endoscopic pyloromyotomy (POP) has been used to improve gastric emptying in selected patients with refractory gastroparesis due to diabetes $[2$, 3] or with gastric outlet obstruction following esophagectomy [4]. To the best of our knowledge, this is the first case in which POP was used to treat PPS.

The patient was an otherwise healthy 18-year-old man with chronic symptoms of gastric outlet obstruction. After a thorough examination, adult idiopathic hypertrophic pyloric stenosis (AIHPS) was diagnosed ( $\bullet$ Fig. 1). He was initially treated several times with injections of onabotulinumtoxinA (Botox, Allergan) into the pylorus, which resulted in transient, dramatic relief of his symptoms.

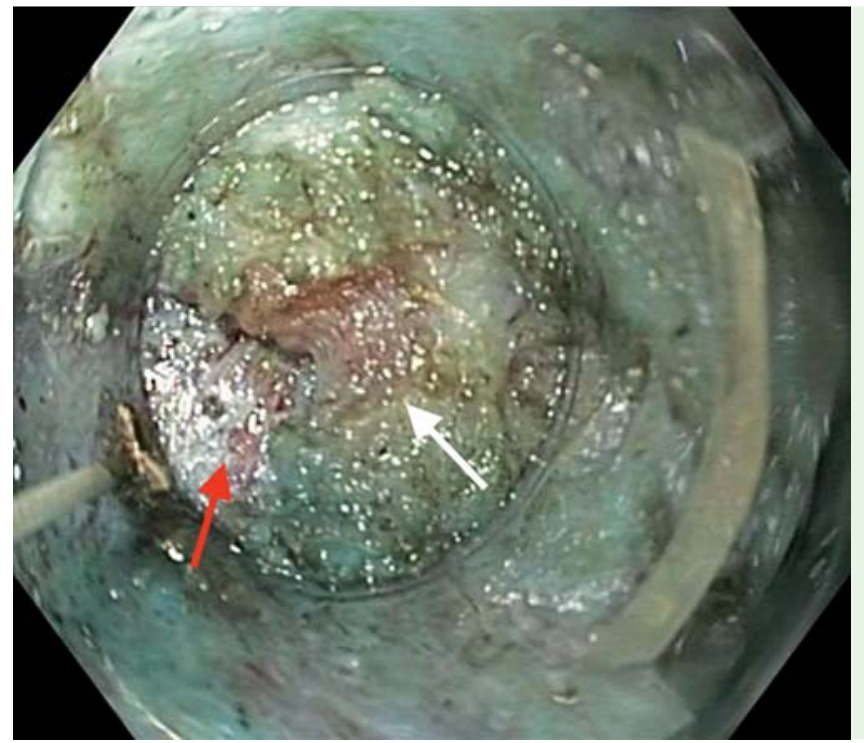

Fig. 2 The circular muscle of the pylorus (white arrow) is dissected until the mucosa of the duodenal bulb (red arrow) is reached.

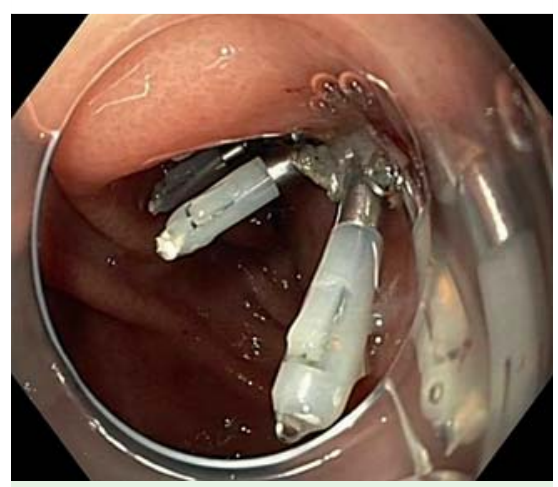

Fig. 3 The mucosal entry is closed with clips.

After the patient had given informed consent, we performed POP while he was under general anesthesia. Prophylactic antibiotics were administered. A posterior myotomy was planned in case the patient were to require a surgical myotomy in the future. After the submucosal injection of $0.9 \% \mathrm{NaCl}$ approximately $5 \mathrm{~cm}$ proximal to the pylorus, a $1-\mathrm{cm}$ incision was made in the mucosa. A submucosal tunnel was created along the posterior gastric wall to the pyloric ring with a Triangle Tip Electrosurgical Knife (KD-640L; Olympus, Tokyo, Japan). The circular muscle of the pylorus was dissected until the mucosa of the duodenal bulb had been reached ( $\mathbf{F i g . 2}$ ). The longitudinal muscular layer was left

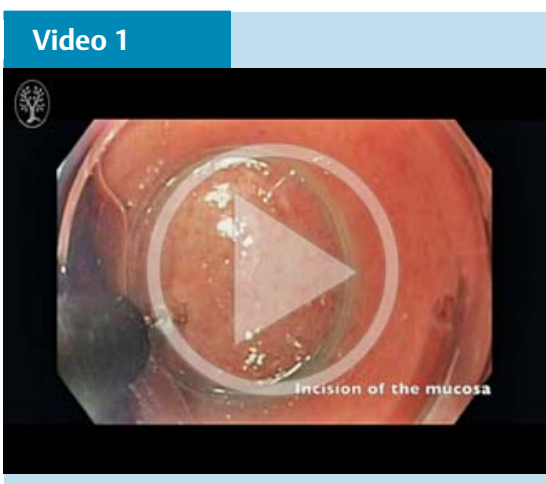

Peroral endoscopic pyloromyotomy for the treatment of primary pyloric stenosis. The steps of the procedure are explained, followed by an overview of the equipment used.

intact. The endoscope was withdrawn, and the mucosal opening was closed with six endoscopic clips (HX-610-090L; Olympus) ( $\bullet$ Fig. 3, $\bullet$ Video 1$)$. The patient was observed overnight while fasting and discharged the next day. No complications developed. At 6-month follow-up, he remained free of symptoms.

In conclusion, POP appears to be a valuable approach that can relieve the symptoms of patients with PPS.

Endoscopy_UCTN_Code_TTT_1AQ_2AG

Competing interests: None 
Khanh Do-Cong Pham ${ }^{1,2}$, Asgaut Viste $^{2,3}$, Aly Dicko ${ }^{3}$, Trygve Hausken ${ }^{1,2}$, Jan Gunnar Hatlebakk ${ }^{1,2}$

${ }^{1}$ Department of Medicine, Haukeland University Hospital, Bergen, Norway

${ }^{2}$ Department of Clinical Medicine, K1, University of Bergen, Norway

${ }^{3}$ Department of Surgery, Haukeland University Hospital, Bergen, Norway

\section{References}

1 Hellan M, Lee T, Lerner T. Diagnosis and therapy of primary hypertrophic pyloric stenosis in adults: case report and review of literature. J Gastrointest Surg 2006; 10: 265 269

2 Khashab MA, Stein E, Clarke JO et al. Gastric peroral endoscopic myotomy for refractory gastroparesis: first human endoscopic pyloromyotomy (with video). Gastrointest Endosc 2013; 78: 764-768

3 Shlomovitz E, Pescarus R, Cassera MA et al. Early human experience with per-oral endoscopic pyloromyotomy (POP). Surg Endosc 2015; 29: 543-551

4 Chung H, Dallemagne B, Perretta S et al. Endoscopic pyloromyotomy for postesophagectomy gastric outlet obstruction. Endoscopy 2014; 46 (Suppl. 01): E345-E346
Bibliography

Dol http://dx.doi.org/

10.1055/s-0034-1393675

Endoscopy 2015; 47: E637-E638

(c) Georg Thieme Verlag KG

Stuttgart · New York

ISSN 0013-726X

\section{Corresponding author}

Khanh Do-Cong Pham, MD

Section of Gastroenterology

Department of Medicine,

Haukeland University Hospital

Jonas Lies vei 65

5051 Bergen

Norway

Fax: +47-55-955000

phamkdc@gmail.com 